\title{
Structure and function of SemiSWEET and SWEET sugar transporters
}

\author{
Liang Feng ${ }^{1}$ and Wolf B. Frommer ${ }^{2}$ \\ ${ }^{1}$ Department of Molecular and Cellular Physiology, 279 Campus Drive, Stanford \\ University School of Medicine, Stanford CA 94305 \\ ${ }^{2}$ Carnegie Institution for Science, Department of Plant Biology, 260 Panama St., \\ Stanford CA 94305, USA
}

Authors for correspondence

Liang Feng, liangf@stanford.edu

Wolf B Frommer wfrommer@ carnegiescience.edu

Abbrev.: THB, triple helix bundle; TM, transmembrane domain. 


\begin{abstract}
Summary
SemiSWEETs and SWEETs emerged as unique sugar transporters. First discovered in plants with the help of fluorescent biosensors, homologs exist in all kingdoms of life. Bacterial and plant homologs transport hexoses and sucrose; animal SWEETs transport glucose. Prokaryotic SemiSWEETs are small and composed of a parallel homodimer of a $\sim 100$ amino acid-long triple helix bundle (THB). Duplicated THBs are fused to create eukaryotic SWEETs in a parallel orientation via an inversion linker helix, producing a similar configuration as in SemiSWEET dimers. Structures of four SemiSWEETs have been resolved in three states: open outside, occluded, and open inside, indicating alternating access. The atomic structures provide a basis for exploring the evolution of structure function relationships in this new class of transporters.
\end{abstract}

Key words: glucose, fructose, hexose, sucrose, carrier, crystal structure 


\section{SWEETs and sugar transport}

Living organisms depend on soluble sugars as the major source of carbon skeletons and energy. The organisms have found ways to facilitate passage of sugars across cellular membranes and to control influx and efflux depending on supply and demand.

Over the past 20 years, many key sugar transporters have been identified from bacteria, fungi, plants and humans (Chen et al., 2015a). These transporters could be categorized into four superfamilies: bacterial PTS (Phosphotransferase) systems, ATP-binding cassette transporters, MFS (major facilitator superfamily) transporters found across all kingdoms and the bacterial and metazoan SSF (sodium-solute symporter family) transporters. The most prominent members of the MFS family include the bacterial lac permease (Chaptal et al., 2011), the yeast HXTs, the human GLUTs and the plant STPs and SUTs (Eom et al., 2015); the best studied sugar transporters in the SSF family are the SGLTs (Wright et al., 2011). Recently, a novel family of sugar transporters was identified, which includes SWEETs and SemiSWEETs. Both belong to the MtN3/saliva clan (CL0141) and their homologs are found in all kingdoms of life (Chen et al., 2010, 2012, 2015b; Lin et al., 2014). The founding members of this family were identified by screening membrane proteins from plants for which no biological function had been assigned using fluorescent sugar sensors. Screens using fluorescent glucose sensors identified the first members (e.g. Arabidopsis SWEET1 and 8 as hexose transporters)(Chen et al., 2010), later, a similar screen using fluorescent sucrose sensors identified members that were able to transport sucrose (Chen 2012, Lin 2014). Homologs able to transport glucose were found also in humans and the worm Caenorhabditis elegans (Chen et al., 2010). The eukaryotic SWEETs all have a predicted topology with seven transmembrane spanning domains which is built from a repeat of three TMs separated by a single TM (Chen et al., 2010) (Figure 1). Bioinformatic studies identified homologs in bacteria (named SemiSWEETs), which are much smaller, with only about 100 amino acids and predicted to contain only one of the repeats (Xuan et al., 2013). SemiSWEETs are widely distributed among prokaryotes including Archaea and Eubacteria, but are sparse, i.e. not all bacteria have a SemiSWEET. A SemiSWEET (LbSemiSWEET from Leptospira (Xu et al., 2014)) has been shown to transport glucose while two members (BjSemiSWEET from Bradyrhizobium (Xuan et al., 2013) and EcSemiSWEET from $E$. coli (Lee et al., 2015)) are able to mediate sucrose transport. A lot of information has been gained into the physiological role of the plant SWEETs, while at present, little is known about animal SWEETs and nothing is known to date about the physiological role of bacterial SemiSWEETs. This article summarizes information on the biological role of SWEETs and focuses on the recent analysis of atomic resolution structures of several SemSWEETs.

\section{Important physiological roles of SWEETs in plants}

Plant genomes typically contain 20 SWEET paralogs, which are differentially expressed, implicating them in a wide range of sugar translocation steps. Arabidopsis SWEETs fall into four subclades and share between 27 and $80 \%$ identity. Clade I, II and IV appear to be predominantly hexose transporters, clade III SWEETs transport predominantly sucrose, although some also can transport hexoses. SWEETs can localize to different cellular compartments, in particular the plasma membrane (e.g. SWEET1, 8, 9, 11,12,15 (Chen et al., 2010, 2012, 2015b; Guan et al., 2008; Lin et al., 2014; Seo et al., 

al., 2013)), and the Golgi (SWEET9, 15(Chen et al., 2015b; Lin et al., 2014)). As common for transporters, which typically are polytopic membrane proteins (type IV multiple-pass $\alpha$-helices), no apparent $\mathrm{N}$-terminal signal sequences for targeting to a specific membrane compartment can be discerned. The existence of transporters from the same family being targeted to different compartments has been found also for other transporters such as peptide transporters (Komarova et al., 2012) and aquaporins (Luu and Maurel, 2013).

A combination of expression analyses, localization studies and analyses of mutants in Arabidopsis has assigned specific roles to individual SWEETs in specific steps in the sugar translocation pathways of plants, specifically sugar efflux in nectar secretion, sucrose efflux from phloem parenchyma cells for phloem loading, seed filling and for pollen nutrition (Table 1). A rather unexpected discovery was that SWEETs play a role in pathogen susceptibility (Chen et al., 2010). A prominent example is the rice locus Xa13 (SWEET11/Os8N3), which is responsible for the recessive rice blight resistance (Chu et al., 2006b; Yang et al., 2006; Yuan et al., 2009). Any of the sucrose-transporting SWEETs in the rice genome can potentially serve as a susceptibility factor (Streubel et al., 2013) when recruited by a TAL effector made in Xanthomonas oryzae oryzae, the causative agent of blight disease. However, when the respective SWEETs become unavailable due to mutations in the SWEET promoter or through RNA interference (Chu et al., 2006a; Yang et al., 2006), sugar supply presumably becomes limiting and the pathogen cannot multiply efficiently. Since pathogens infect plants to reproduce, and since they require nutrients for efficient reproduction, this may be a general mechanism. This hypothesis is supported by the observation that SWEETs are also important for susceptibility to cassava blight (Cohn et al., 2014).

\section{Physiological role of SWEETs in animals and humans}

To date, and despite the apparent potential for affecting sugar homeostasis in animals and humans, very little is known about the physiological role of SWEETs in animals. Although animal genomes including human typically contain only a single SWEET gene, a major exception is $C$. elegans, which contains 7 SWEET paralogs. Both the human and one of the $C$. elegans SWEETs mediate glucose transport. Their broad expression patterns implicate them as fundamental sugar transporters in animal and human physiology. The first identified member of this family in animals was the Drosophila gene saliva (slv), now recognized as DmSWEET1 from sequence similarity. Overexpression of slv affected axonal growth and guidance (Nicolai et al., 2003). It remains unclear whether this is a specific effect, or whether this is due to interference with polarized sugar translocation. In plants, ectopic expression of SWEETs negatively impacts growth, indicating that uncontrolled overexpression leads to toxicity possibly by creating leaks from cells that normally do not efflux sugars (Eom et al., 2015). However, the characterization of mutations in the sea squirt SWEET gene (Ci-RGA) caused defects early in development, underlining the importance of SWEETs in metazoa (Hamada et al., 2005). Given the importance of sugar homeostasis in humans, it will be important to systematically study the physiological role of SWEETs and their regulation in different states including metabolic diseases.

\section{SemiSWEET structures}


Recent breakthroughs in the structural determination of four SemiSWEET homologs revealed the architecture of SemiSWEET, captured three conformational states and provided rich structural insights into the transport mechanism (Lee et al., 2015; Wang et al., 2014; Xu et al., 2014).

This burst of structures started in 2014 when VsSemiSWEET (from Vibrio sp. N418) and LbSemiSWEET (from Leptospira biflexa serovar Patoc) were determined at $1.7 \AA$ and $2.4 \AA$ resolution, respectively (Xu et al., 2014). VsSemiSWEET turned out to be in an outward open state while LbSemiSWEET was in an occluded state. Then the structure of TySemiSWEET (from T. yellowstonii DSM 11347) in an occluded state (Wang et al., 2014), and structures of EcSemiSWEET (from E. coli) in both inward open and outward open states (Lee et al., 2015) were also determined. All these structures were determined using crystals grown in lipid cubic phase, which apparently was essential to obtain high quality crystals of SemiSWEETs.

These structures unambiguously revealed the architecture of SemiSWEET (Table 2). The basic structural unit of SemiSWEET is a triple-helix-bundle (THB) formed by the three transmembrane (TM) helices of a protomer. These three TMs are arranged in a 1-3-2 manner (Figure 1). As a result, there is intimate interaction between TM2 - TM3, and TM1 - TM3 while TM1 makes little direct contact with TM2. Compatible with this arrangement, the loop L1-2 connecting TM1 and TM2 is much more extended than loop L2-3 between TM2 and TM3. The 'positive-inside' rule predicts that the L1-2 loop with several positively charged residues locates to the intracellular side of the transporter. It is noteworthy that a 1-3-2 THB was also thought to be the basic structural unit of the MFS superfamily (Yan 2013), even though SemiSWEET and MFS are unrelated in sequence or overall structure. The THB within the protomer forms a concaved, but not enclosed surface, so it cannot form a functional transporter by itself.

Two copies of protomers come together to form a dimer in the crystal (Lee et al., 2015; Wang et al., 2014; Xu et al., 2014). The same dimeric architecture was observed in all four SemiSWEETs with highly diverged sequences, which strongly suggest that the dimer is the fundamental structure of SemiSWEETs. Several observations further support the dimer as a common functional unit: 1) a central putative transport route is formed at the dimer interface, which is compatible with the sugar transport function of SemiSWEET; 2) cross-linking BjSemiSWEET in solution showed dimer formation (Xu et al., 2014); and 3) crystal packing indicates dimer formation within lipid bilayers of lipid cubic phase (LCP). The dimer interface is mainly mediated by TM1-TM2 crossprotomer interactions. In addition, loop L1-2 also contributes significantly to the interface in occluded and outward open states, whereas loop L2-3 forms part of the interface in inward open and occluded state. The interface between the protomers buries a large surface area (in the range of $2000 \AA^{2}$ ), which further supports the biological relevance of dimeric architecture.

Though heptahelical vitamin transporter PnuC and G-protein coupled receptors were proposed to be related to SWEETs based on remote sequence pattern similarity and positions of TM helices (Jaehme et al., 2014; Jaehme and Slotboom 2015; Yee et al., 2013), their topologies differ from that of SemiSWEET dimer. TMs of GPCR and PnuC core are connected sequentially with each TM interacting with the next one in sequence, which is in contrast to 1-3-2 connectivity in THB of SemiSWEET. These differences 

they diverge from a common ancient ancestral building unit remains to be determined.

\section{Transport pathway and putative substrate binding pocket}

A fundamental question about any particular transporter is how it forms a selective transport route for its substrates within the membrane. The availability of four SemiSWEET structures enables the identification and a close examination of the transport pathway that is formed around the two-fold symmetry axis. The transport route is shielded by TMs from the membrane and only accessible either from extracellular side in the outward open state or from intracellular side in the inward open state. The hydrophobicity of the transport route differs among different SemiSWEETs, which might reflect their diverse physiological properties.

Though none of the available SemiSWEET structures are bound with known substrates, all the structures point to the same putative substrate binding pocket right above the center of the protein. In both LbSemiSWEET and TySemiSWEET, a large enclosed cavity was observed in the crystal structure that is sufficient to accommodate a sugar molecule (Wang et al., 2014; Xu et al., 2014). In both cases, extra non-protein densities of unknown identity were observed, providing further support for the putative substratebinding pocket. The pocket size of TySemiSWEET differs from that of LbSemiSWEET in an intriguing way: the pocket of TySemiSWEET is sufficiently large to accommodate a disaccharide, while the pocket of LbSemiSWEET can only hold a monosaccharide but not a disaccharide. Interestingly, the extra density in the TySemiSWEET pocket is also much larger than that of LbSemiSWEET. This raises the possibility that pocket size plays important roles in determining substrate specificity (Wang et al., 2014), which presents a testable hypothesis for functional studies. In VsSemiSWEET and EcSemiSWEET, a PEG400 molecule and the head group of a monoolein lipid molecule, respectively, were found in the corresponding putative substrate-binding pocket. PEG and lipid molecules are present at very high concentration during crystallization and are frequently found in protein crystal structures. Their location is in line with a small molecule-accommodating pocket (Lee et al., 2015; Xu et al., 2014).

Among all the residues lining the putative substrate-binding pocket, Trp48 and Asp64 (numbering in LbSemiSWEET) are the most conserved, essentially invariant among SemiSWEETs (68 diverse SemiSWEET sequences analyzed). In a SemiSWEET dimer, these two pairs of residues sit at similar levels and surround the extra densities in the cavities of LbSemiSWEET and TySemiSWEET (Wang et al., 2014; Xu et al., 2014). They are poised to make contact with any putative sugar molecules in the pocket through either hydrogen bonds or aromatic ring stacking interactions. Their important roles in transport are confirmed by mutagenesis studies in LbSemiSWEET and EcSemiSWEET. Conversion of Asn to Ala abolished the transport activities. The effect of Trp mutations is more complex: W64A in LbSemiSWEET impacted glucose transport activity to background levels, while the corresponding mutations in EcSemiSWEET increased sucrose transport activity (Lee et al., 2015; Xu et al., 2014). Given that transport activities and substrates are different for LbSemiSWEET and EcSemiSWEET and that the exact binding sites of the sugars are not available so far, it is still unclear what causes these 

critical roles of Asn and Trp in sugar transport. It is also interesting to note that the symmetrical binding pocket and the asymmetrical substrate create a geometrical mismatch. Structures of the SemiSWEET in substrate-bound forms are needed to reveal how SemiSWEET functions. Current SemiSWEET structures suggest a single substratebinding site within the protein. To translocate the sugar across the membrane, SemiSWEET needs to go through conformational change cycles to translocate the substrate.

\section{Conformation states and transport cycle}

SemiSWEET structures in different conformational states are fully compatible with an alternating access model (Lee et al., 2015; Wang et al., 2014; Xu et al., 2014), in which the substrate-binding pocket alternatively exposes to either side of the membrane. VsSemiSWEET and one form of EcSemiSWEET were captured in an outward open formation, with a large solvent access route from the extracellular surface all the way to the putative substrate-binding pocket. LbSemiSWEET and TySemiSWEET were crystalized in an occluded state, with a cavity completely enclosed by the protein. One form of EcSemiSWEET showed an inward open conformation, with the putative substrate-binding pocket accessible from the intracellular side. Although comparing different conformations from different protein homologs inevitably faces the caveat of structural divergence caused by sequence differences, it provides an overall picture of the transport cycle and insights into transport mechanism.

Three structural elements at the dimer interface play important roles in the transport cycle (Lee et al., 2015; Wang et al., 2014; Xu et al., 2014) (Figure 1): (i) The PQ motif on TM1, which is highly conserved in SemiSWEET and the signature motif in the related PQ loop family protein. In the occluded conformation and outward open conformation, side chain of glutamine makes the cross-protomer hydrogen bonds with backbone amides in loop L1-2, which helps to bring the two L1-2 in close proximity to seal the intracellular side. In the inward open conformation, these hydrogen bonds were disrupted to open up the intracellular access route. Therefore, the PQ motif is designated as part of the intracellular gate. (ii) D-Y pairs on the loop L2-3. Aspartate forms cross-protomer hydrogen bonds with tyrosine and shields the putative binding pocket from the extracellular solution in the occluded and inward open conformations (Figure 2). In the outward open state, aspartate and tyrosine are separated away to expose the binding pocket to the outside solution. D-Y pairs thus form the extracellular gate. (iii) The pocket lining Trp (as W48 in LbSemiSWEET) of one protomer forms hydrogen bond with Thr on TM1 (as T13 in LbSemiSWEET) of the other protomer in LbSemiSWEET, TySemiSWEET and EcSemiSWEET. Given the important role of Trp in substrate binding and TM1 conformation changes during the transport cycle, this interaction indicates a possible link between substrate binding and the conformational transition among states.

Among different states, TM2 and TM3 within a protomer remain together like a rigid body. The degree of TM1 movement among different conformations is less clear as TM1 of VsSemiSWEET and EcSemiSWEET in the outward open state adopts different tilt angle relative to TM2/TM3. Nonetheless, TM1 of EcSemiSWEET forms a $\sim 30^{\circ}$ kink around the conserved PQ motif when transition from outward open to an inward open 
state (Lee et al., 2015). These structures suggest a minimally four-state alternating access cycle, which is accompanied by sequential formation and breakage of intracellular and extracellular gate to allow substrate transport (Figure 3).

\section{Concluding remarks}

The recent identification and functional characterization of SWEET proteins and structural elucidation of their bacterial homologs, SemiSWEETs, have greatly advanced our understanding of the function and transport mechanism of this important protein family. Despite of these rapid progresses, many open questions remain regarding the transport cycle, the transport mechanism and the physiological roles especially of the bacterial and animal SWEETs (see Box 1).

A first question is what drives the conformational transitions during the transport cycle. It has been speculated that SWEET might function as facilitative uniporter due to low affinity and relative $\mathrm{pH}$ independence in vivo. Functional characterizations of SWEET/SemiSWEET in vitro would help to delineate the driving force of the transport process.

At the atomic structure level, high-resolution structures of SemiSWEET from several species have captured three different conformational states that could be used to construct an alternating access model. However, lack of a complete set of conformational states from a single SemiSWEET hinders the dissection of conformational changes during the transport cycle. Furthermore, SemiSWEET structures in complex with substrate sugars are in high demand in order to elucidate the mechanism of substrate recognition and specificity. Besides bacterial SemiSWEET structures, no structure of eukaryotic SWEET has been reported yet. It has been proposed that SWEET and SemiSWEET dimer may be structurally related based on the existence of the dual THB in SWEET and functional conservations of key residues (Xu et al., 2014). However, it is not really feasible to build a structural model of SWEET by homology modeling due to distant sequence similarities between SemiSWEET and eukaryotic SWEET and the existence of the inversion linker TM4 in the heptahelical SWEET. To elucidate the transport mechanism of eukaryotic SWEET and to understand the relationship between SemiSWEETs and eukaryotic SWEETs, it is essential to obtain high-resolution structures of eukaryotic SWEETs, which still remains challenging in the field.

Beyond static structural snapshots, it is important to study dynamics of SWEET/SemiSWEET in order to understand their transport mechanism. Spectroscopic methods such as single molecule FRET, DEER (double electron-electron resonance) and NMR will provide insights into dynamic properties, conformational distributions, as well as local and global conformational changes of SWEET/SemiSWEET proteins during the transport cycle. These studies will help to elucidate how the transport process is coupled to the conformational transitions in SWEET/SemiSWEET.

Structural and functional characterizations have provided hints about the relationship between SWEET and SemiSWEET. The evolution of transporters from smaller units, most likely through gene duplication of an ancestral simpler structure, as appears to be the case for SWEETs which may have evolved from SemiSWEETs, is not uncommon (Keller et al., 2014). Unusual is the parallel orientation of the THB in SWEETs, which required the insertion of an inversion linker helix. The present analyses show a clear 
distinction in that prokaryotes contain the ancestral form, while eukaryotes in all kingdoms have the fused form. Phylogenetic studies may help to identify the origin of the two THBs in SWEETs from either a single gene duplication event or multiple sources and steps.

\section{Acknowledgements}

Original research has been supported by grants from the Department of Energy (DEFG02-04ER15542), the National Science Foundation (IOS-1258018) and the Bill and Melinda Gates Foundation to W.F and by Stanford University, the Harold and Leila Y. Mathers Charitable Foundation and Alfred P. Sloan Foundation to L.F. We thank Frommer lab members and Feng lab members for stimulating discussions. 


\section{References}

Antony, G., Zhou, J., Huang, S., Li, T., Liu, B., White, F., and Yang, B. (2010). Rice xa13 recessive resistance to bacterial blight is defeated by induction of the disease susceptibility gene Os-11N3. Plant Cell 22, 3864-3876.

Chaptal, V., Kwon, S., Sawaya, M.R., Guan, L., Kaback, H.R., and Abramson, J. (2011). Crystal structure of lactose permease in complex with an affinity inactivator yields unique insight into sugar recognition. Proc. Natl. Acad. Sci. U. S. A. 108, 9361-9366.

Chardon, F., Bedu, M., Calenge, F., Klemens, P.A., Spinner, L., Clement, G., Chietera, G., Leran, S., Ferrand, M., Lacombe, B., et al. (2013). Leaf Fructose Content Is Controlled by the Vacuolar Transporter SWEET17 in Arabidopsis. Curr. Biol. 23, 697702.

Chen, L.Q., Hou, B.H., Lalonde, S., Takanaga, H., Hartung, M.L., Qu, X.Q., Guo, W.J., Kim, J.G., Underwood, W., Chaudhuri, B., et al. (2010). Sugar transporters for intercellular exchange and nutrition of pathogens. Nature 468, 527-532.

Chen, L.Q., Qu, X.Q., Hou, B.H., Sosso, D., Osorio, S., Fernie, A.R., and Frommer, W.B. (2012). Sucrose efflux mediated by SWEET proteins as a key step for phloem transport. Science 335, 207-211.

Chen, L.-Q., Cheung, L.S., Feng, L., Tanner, W., and FROMMER, W.B. (2015a). Transport of Sugars. Annu. Rev. Biochem. 84, 1-20.

Chen, L.Q., Qu, X.Q., Sosso, D., McFarlane, H.E., Samuels, A.L., and Frommer, W.B. (2015b). Embryo nutrition by a cascade of sequentially expressed sucrose transporters in the seed coat. Plant Cell 27, 607-619.

Cohn, M., Bart, R.S., Shybut, M., Dahlbeck, D., Gomez, M., Morbitzer, R., Hou, B.-H., Frommer, W.B., Lahaye, T., and Staskawicz, B.J. (2014). Xanthomonas axonopodis virulence is promoted by a Transcription Activator-Like Effector-mediated induction of a SWEET sugar transporter in cassava. Mol. Plant-Microbe Interact. MPMI 27, 11861198.

Eom, J.S., Chen, L.-Q., Sosso, D., Lin, I.W., Qu, X.-Q., and Frommer, W.B. (2015). The SWEET-SUT couple, transporters mediating sucrose transfer between neighboring cells. Curr. Opin. Plant Biol. submitted.

Guan, Y.F., Huang, X.Y., Zhu, J., Gao, J.F., Zhang, H.X., and Yang, Z.N. (2008). RUPTURED POLLEN GRAIN1, a member of the MtN3/saliva gene family, is crucial for exine pattern formation and cell integrity of microspores in Arabidopsis. Plant Physiol. 147, 852-863.

Guo, W.-J., Nagy, R., Chen, H.-Y., Pfrunder, S., Yu, Y.-C., Santelia, D., Frommer, W.B., and Martinoia, E. (2014). SWEET17, a facilitative transporter, mediates fructose 
transport across the tonoplast of Arabidopsis roots and leaves. Plant Physiol. 164, 777789.

Jaehme, M., Guskov, A., and Slotboom, D.J. (2014). Crystal structure of the vitamin B3 transporter PnuC, a full-length SWEET homolog. Nat. Struct. Mol. Biol. 21, 1013-1015.

Jaehme M, Slotboom DJ. (2015) Structure, function, evolution and application of bacterial Pnu-type vitamin transporters. Biol Chem. 2015 Feb 24. doi: 10.1515/hsz-20140113. [Epub ahead of print]

Keller, R., Ziegler, C., and Schneider, D. (2014). When two turn into one: evolution of membrane transporters from half modules. Biol. Chem. 395, 1379-1388.

Klemens, P.A., Patzke, K., Deitmer, J.W., Spinner, L., Le Hir, R., Bellini, C., Bedu, M., Chardon, F., Krapp, A., and Neuhaus, E. (2013). Overexpression of the vacuolar sugar carrier AtSWEET16 modifies germination, growth and stress tolerance in Arabidopsis thaliana. Plant Physiol. 63, 1338-1352.

Komarova, N.Y., Meier, S., Meier, A., Grotemeyer, M.S., and Rentsch, D. (2012). Determinants for Arabidopsis peptide transporter targeting to the tonoplast or plasma membrane. Traffic Cph. Den. 13, 1090-1105.

Lee, Y., Nishizawa, T., Yamashita, K., Ishitani, R., and Nureki, O. (2015). Structural basis for the facilitative diffusion mechanism by SemiSWEET transporter. Nat. Commun. 6,6112 .

Lin, I.W., Sosso, D., Chen, L.Q., Gase, K., Kim, S.G., Kessler, D., Klinkenberg, P.M., Gorder, M.K., Hou, B.H., Qu, X.Q., et al. (2014). Nectar secretion requires sucrose phosphate synthases and the sugar transporter SWEET9. Nature 508, 546-549.

Luu, D.-T., and Maurel, C. (2013). Aquaporin trafficking in plant cells: an emerging membrane-protein model. Traffic Cph. Den. 14, 629-635.

Nicolai, M., Lasbleiz, C., and Dura, J.M. (2003). Gain-of-function screen identifies a role of the Src64 oncogene in Drosophila mushroom body development. J. Neurobiol. 57, 291-302.

Seo, P.J., Park, J.M., Kang, S.K., Kim, S.G., and Park, C.M. (2011). An Arabidopsis senescence-associated protein SAG29 regulates cell viability under high salinity. Planta $233,189-200$.

Streubel, J., Pesce, C., Hutin, M., Koebnik, R., Boch, J., and Szurek, B. (2013). Five phylogenetically close rice SWEET genes confer TAL effector-mediated susceptibility to Xanthomonas oryzae pv. oryzae. New Phytol. 200, 808-819.

Sun, M.X., Huang, X.Y., Yang, J., Guan, Y.F., and Yang, Z.N. (2013). Arabidopsis RPG1 is important for primexine deposition and functions redundantly with RPG2 for plant fertility at the late reproductive stage. Plant Reprod. 26, 83-91. 
Wang, J., Yan, C., Li, Y., Hirata, K., Yamamoto, M., Yan, N., and Hu, Q. (2014). Crystal structure of a bacterial homologue of SWEET transporters. Cell Res. 24, 1486-1489.

Wright, E.M., Loo, D.D.F., and Hirayama, B.A. (2011). Biology of human sodium glucose transporters. Physiol. Rev. 91, 733-794.

Xu, Y., Tao, Y., Cheung, L.S., Fan, C., Chen, L.-Q., Xu, S., Perry, K., Frommer, W.B., and Feng, L. (2014). Structures of bacterial homologues of SWEET transporters in two distinct conformations. Nature 515, 448-452.

Xuan, Y.H., Hu, Y.B., Chen, L.Q., Sosso, D., Ducat, D.C., Hou, B.H., and Frommer, W.B. (2013). Functional role of oligomerization for bacterial and plant SWEET sugar transporter family. Proc. Natl. Acad. Sci. USA 110, E3685-E3694.

Yan, N. (2013) Structural advances for the major facilitator superfamily (MFS) transporters. Trends Biochem Sci. 38, 151-9.

Yee, D.C., Shlykov, M.A., Västermark, Å., Reddy, V.S., Arora, S., Sun, E.I., and Saier, M.H. (2013). The transporter-opsin-G protein-coupled receptor (TOG) superfamily.

FEBS J. 280, 5780-5800. 


\section{Legends to Figures}

Figure 1. SemiSWEET and SWEET topology. (A) SemiSWEET is composed of a simple 1-3-2 triple helix bundle (THB). (B) SWEET contains two THBs that are connected by an inversion linker helix.

A

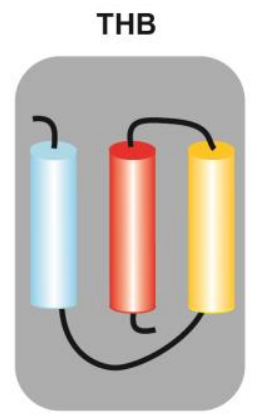

SemiSWEET
B

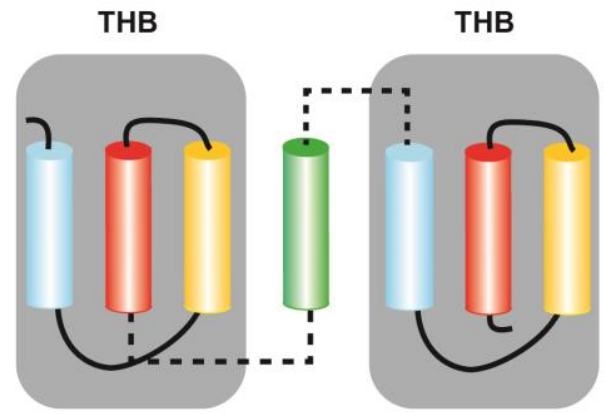

SWEET 
Figure 2: Interface gates. (A) D-Y pairs form the extracellular gate. (B) The glutamine of PQ motif makes cross-protomer hydrogen bonds with L1-2 loop and is part of the intracellular gate. (C) Threonine on TM1 makes hydrogen bond with tryptophan in the substrate-binding pocket on the other protomer, which is proposed to play an important role in the transport cycle.

A

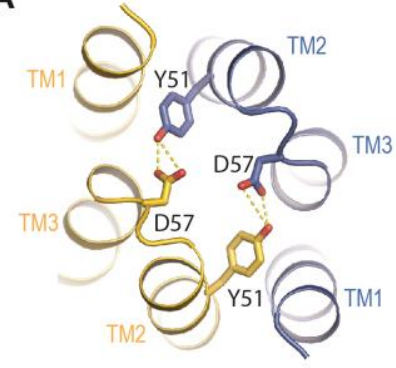

Extracellular gate
B

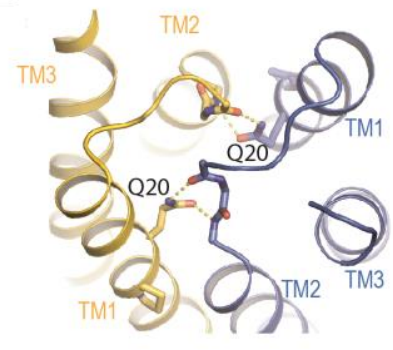

Intracellular gate part
C

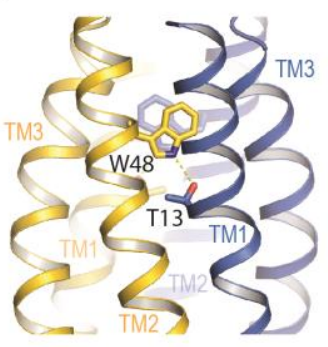

T-W pair 
Figure 3. Structures of SemiSWEET and the proposed alternating access transport model. Structures of SemiSWEETs in the outward open, occluded and inward open conformational states from LbSemiSWEET, VsSemiSWEET, TySemiSWEET and EcSemiSWEET are shown in ribbon representation. Two protomers are colored in yellow and blue respectively. Slab views of the SemiSWEETs, colored by electrostatic potential, are shown below the corresponding ribbon representations. In the proposed transport cycle, SemiSWEET undergoes conformational changes to alternatively expose substrate to either side of the membrane through at least four major states: outward open, occluded (bound), inward open and occluded (empty).
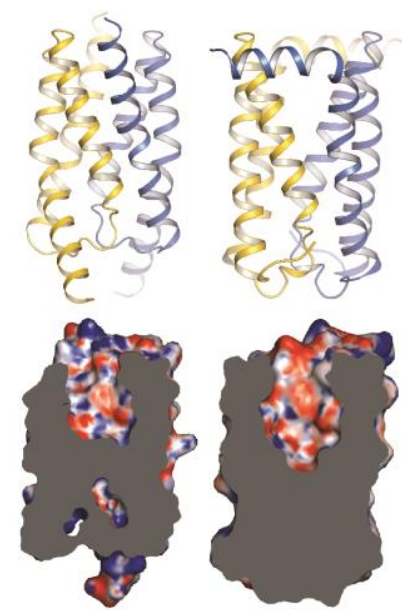

EcSemiSWEET

VsSemiSWEET

Outward open
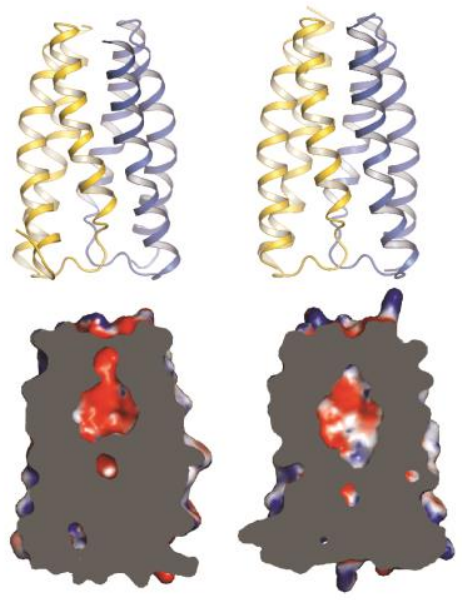

LbSemiSWEET

TySemiSWEET

Occluded
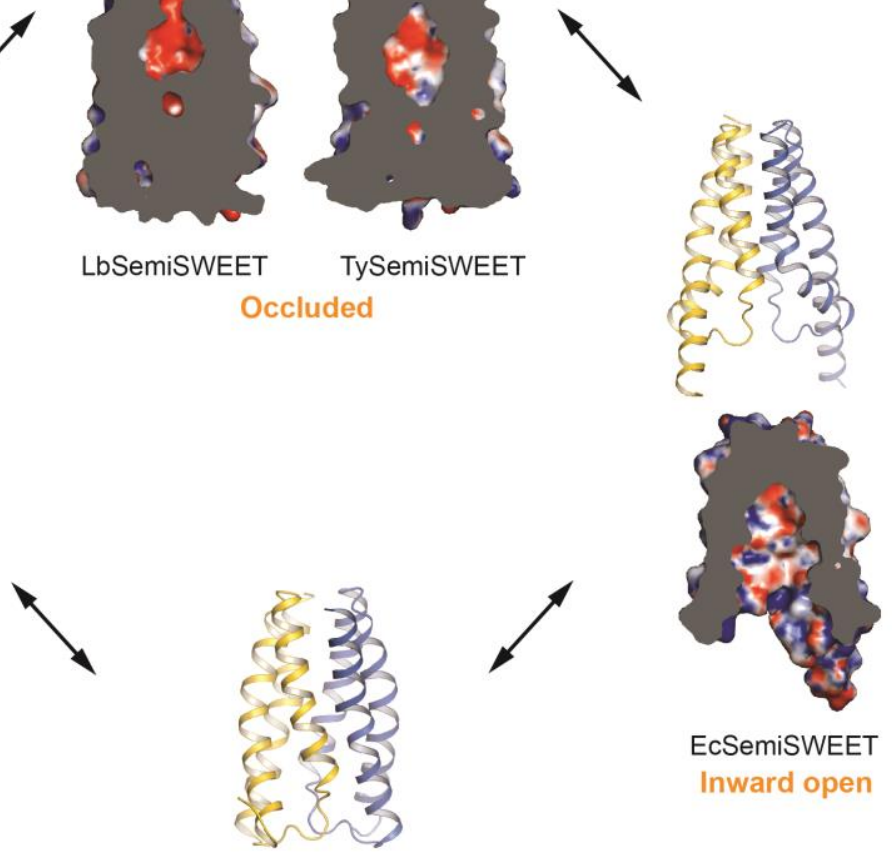

EcSemiSWEET

Inward open

Occluded 


\section{Legends to Tables}

Table 1. Physiological role of SWEETs

\begin{tabular}{|c|c|c|c|}
\hline gene & species & role & reference \\
\hline SWEET8 & Arabidopsis & Pollen development & (Sun et al., 2013) \\
\hline SWEET9 & $\begin{array}{l}\text { Arabidopsis, } \\
\text { Brassica, } \\
\text { Nicotiana }\end{array}$ & Nectar secretion & (Lin et al., 2014) \\
\hline SWEET11,12 & Arabidopsis & $\begin{array}{l}\text { Cellular efflux in leaves for phloem } \\
\text { loading, seed coat/endosperm efflux } \\
\text { for seed filling }\end{array}$ & (Chen et al., 2015b) \\
\hline SWEET13 & Arabidopsis & Pollen development & (Sun et al., 2013) \\
\hline SWEET15 & Arabidopsis & Seed coat efflux, seed filling & (Chen et al., 2015b) \\
\hline SWEET16 & Arabidopsis & Freezing tolerance & $\begin{array}{l}\text { (Klemens et al., } \\
2013 \text { ) }\end{array}$ \\
\hline SWEET17 & Arabidopsis & Fructose content of vacuoles & $\begin{array}{l}\text { (Chardon et al., } \\
\text { 2013) }\end{array}$ \\
\hline $\begin{array}{l}\text { Clade III } \\
\text { SWEETs }\end{array}$ & Rice, Cassava & Pathogen resistance & $\begin{array}{l}\text { Antony et al., } \\
\text { 2010; Chen et al., } \\
\text { 2010; Cohn et al., } \\
\text { 2014) }\end{array}$ \\
\hline
\end{tabular}

Table 2. List of characterized SemiSWEETs

\begin{tabular}{|l|l|l|l|l|l|l|}
\hline BWSemiSWEET & NP_773100.1 & sucrose & ND & ND & $\begin{array}{l}\text { (Xuan et al., } \\
2013)\end{array}$ \\
\hline LbSemiSWEET & B0SR19 & glucose & 4QNC & occluded & $\begin{array}{l}\text { (Xu et al., } \\
2014)\end{array}$ \\
\hline VsSemiSWEET & F9RBV9 & ND & 4QND & Outside open & $\begin{array}{l}\text { (Xu et al., } \\
2014)\end{array}$ \\
\hline TySemiSWEET & B5YGD6 & ND & 4RNG & Occluded & $\begin{array}{l}\text { (Wang et al., } \\
2014)\end{array}$ \\
\hline EcSemiSWEET & T8UDF6 & $\begin{array}{l}\text { Sucrose, } \\
\text { probably not } \\
\text { physiological }\end{array}$ & $\begin{array}{l}\text { 4X5M } \\
\text { 4X5N }\end{array}$ & $\begin{array}{l}\text { Outward open } \\
\text { Inward open }\end{array}$ & $\begin{array}{l}\text { (Lee et al., } \\
2015)\end{array}$ \\
\hline
\end{tabular}




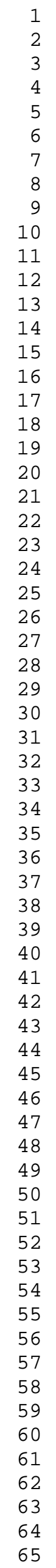




\section{Box 1. Outstanding questions}

- A complete transport cycle: missing so far are SemiSWEET structures in occluded bound and all conformations from the same protein.

- Dynamic analyses of structural rearrangements, e.g. by NMR studies.

- Proof of the actual transport mechanism

- Structures of the eukaryotic SWEETs

- Detailed information on the binding site and the selectivity

- Detailed information on the oligomerization state and dynamics of SWEETs

- Data on the transcriptional and posttranslational regulation of transport

- Physiological role(s) of prokaryotic SemiSWEETs and eukaryotic SWEETs including from humans 
A

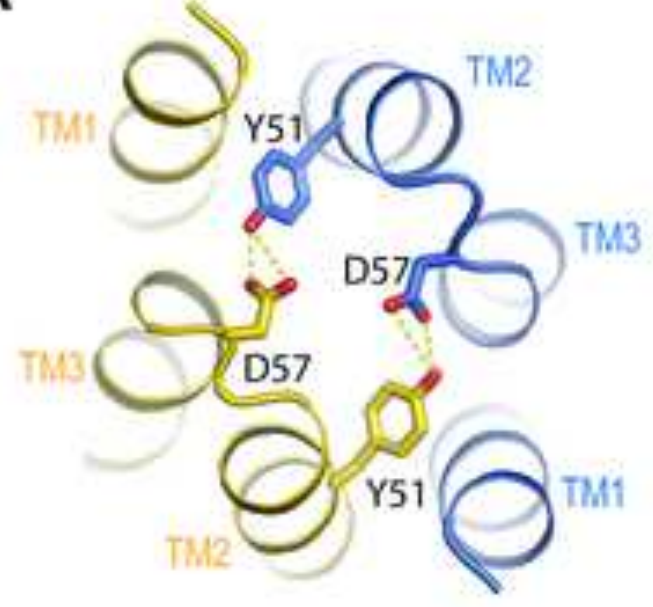

Extracellular gate
B

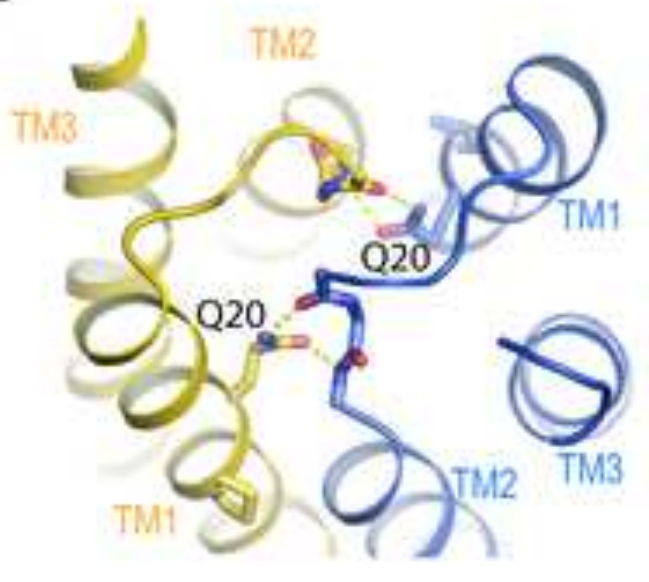

Intracellular gate part c

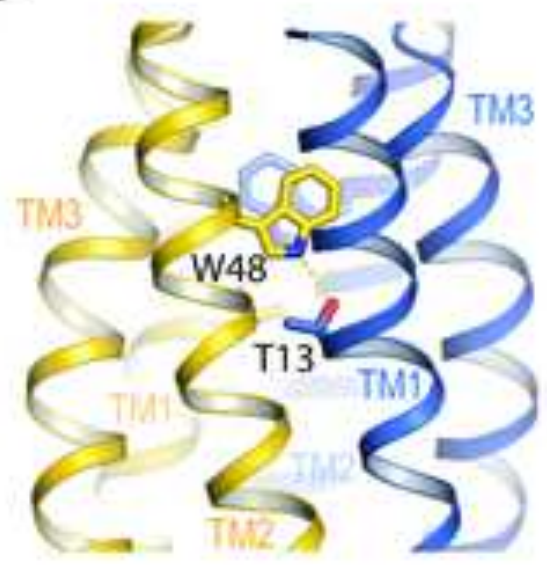

T-W pair 


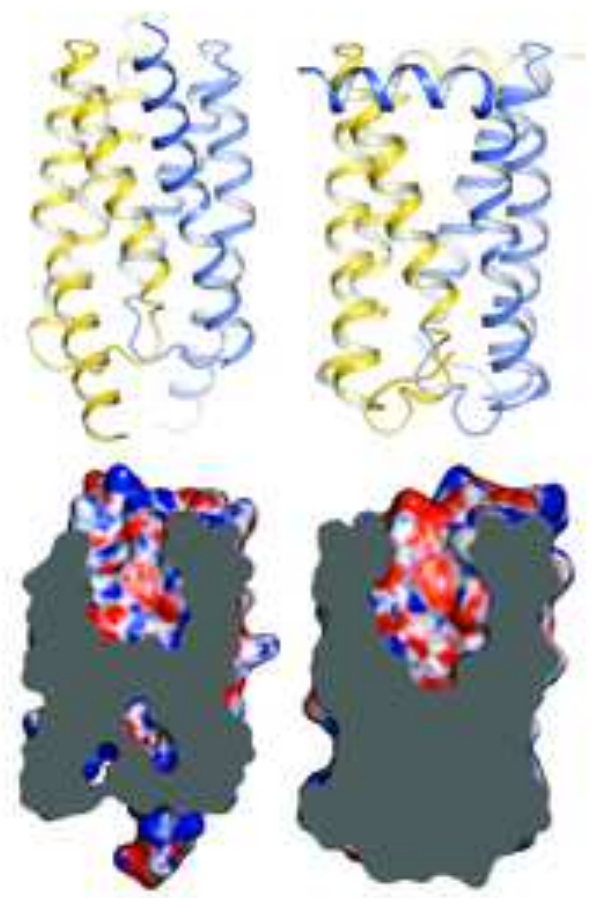

ECSemiSWEET VsSemiSWEET

Outward open
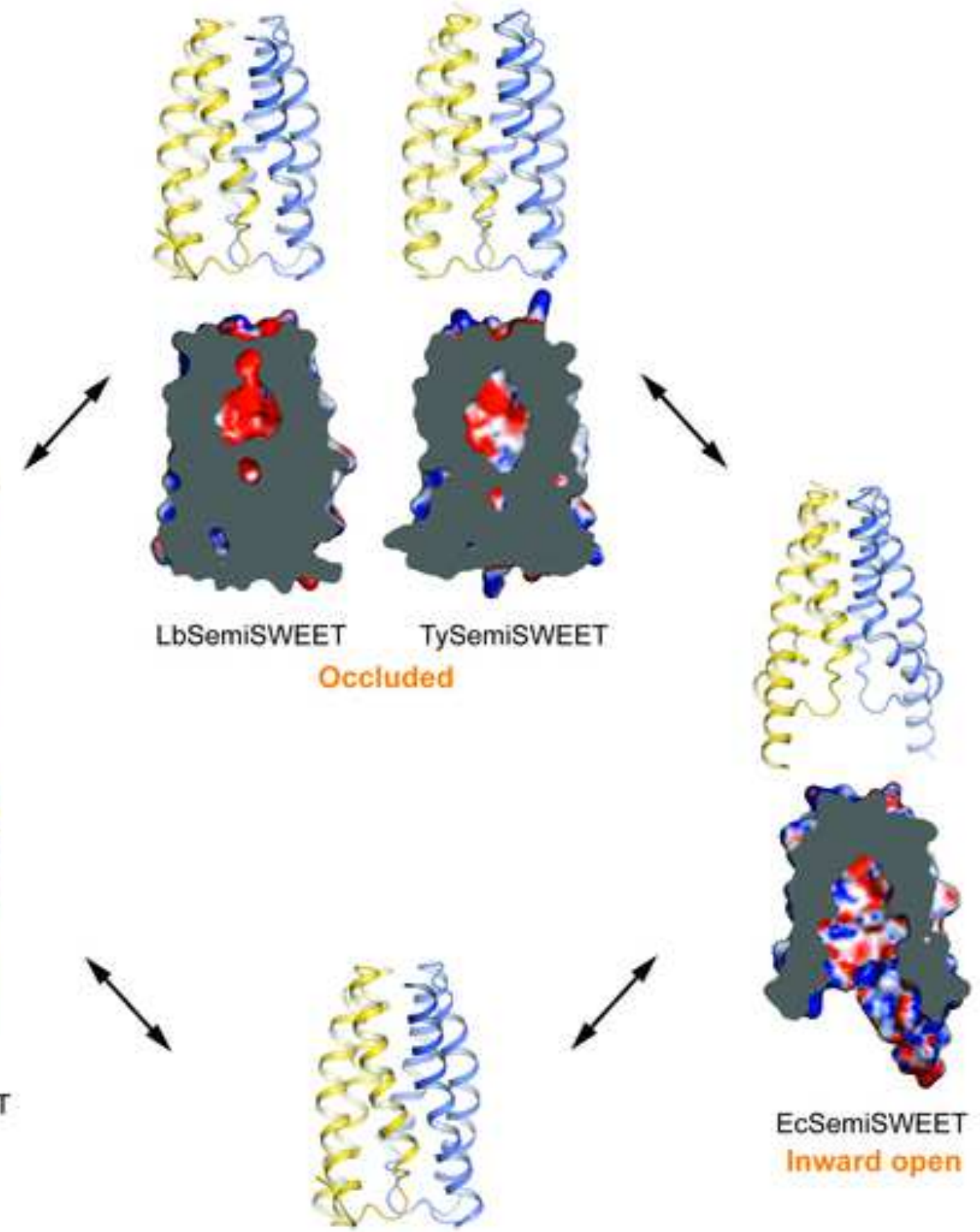

Occluded 